\title{
Adaptive Generalized Synchronization of Drive-Response Neural Networks with Time-Varying Delay
}

\author{
Lirong Zhu \\ Chengyi University College, Jimei University, Xiamen, China \\ Email: 57636934@qq.com
}

How to cite this paper: Zhu, L.R. (2022) Adaptive Generalized Synchronization of Drive-Response Neural Networks with TimeVarying Delay. Applied Mathematics, 13, 19-26.

https://doi.org/10.4236/am.2022.131002

Received: December 13, 2021

Accepted: January 4, 2022

Published: January 7, 2022

Copyright $\odot 2022$ by author(s) and Scientific Research Publishing Inc. This work is licensed under the Creative Commons Attribution International License (CC BY 4.0).

http://creativecommons.org/licenses/by/4.0/

\begin{abstract}
This paper studies the generalized synchronization of a class of drive-response neural networks with time-varying delay. When the topological structures of the drive-response neural networks are known, by designing an appropriate nonlinear adaptive controller, the generalized synchronization of these two networks is obtained based on Lyapunov stability theory and LaSalle's invariance principle.
\end{abstract}

\section{Keywords}

Generalized Synchronization, Drive-Response Neural Network, Time-Varying Delay, Adaptive Controller

\section{Introduction}

There are various complex networks in nature and human society, such as transportation network, biological network, social relationship network, neural network and so on [1] [2] [3]. In recent years, complex networks have attracted the attention of scholars in many fields including biology, engineering, economics, neuroscience, mathematics, physics.

They have been become a research hotspot in academic circles, many interesting and valuable results are obtained [4] [5] [6] [7]. Many studies have been devoted to the synchronization of complex dynamic networks due to its wide application in the real world [8] [9] [10] [11]. Synchronization is a collective phenomenon and behavior, its principle can be used to promote social production and human activities, for instance the security of communication, the development of laser equipment and nuclear magnetic resonance instrument [12]. 
Therefore, the research on synchronization of complex dynamic networks has very important practical significance.

The consistency problem of all nodes in a complex network is internal synchronization. Various synchronization methods have been proposed, such as adaptive control [13] [14] [15], impulse control [16] [17], pinning control [18] [19]. In fact, synchronization can also be realized between two networks, that is, external synchronization, including complete synchronization [20], projective synchronization [21] [22], generalized synchronization [23] [24] [25]. The projective synchronization of two complex networks with the same node dynamics is studied in the literature [22]. By designing a nonlinear controller, the generalized synchronization of two complex networks with different node dynamics was realized, but the time delay was not considered [24]. In [25], the generalized synchronization for two complex networks with time-varying delay coupling was investigated.

Neural networks, as a special kind of complex networks, have been received considerable attention because of its potential application in neurophysiology, automatic control, image processing. The synchronization of two neural networks with time delay was studied in the literature [26]. The projective synchronization of neural networks without time delay was obtained in [21]. The literature [27] realized the projective synchronization of a class of neural networks by using adaptive feedback control method.

However, there are few studies on the generalized synchronization of a class of neural networks with time-varying delay. This paper is concerned with the problem of generalized synchronization for drive-response neural networks with timevarying delay. If the topological structures of the systems are known, by designing an appropriate nonlinear adaptive controller, the generalized synchronization between these two networks can be achieved based on Lyapunov stability theory and LaSalle's invariance principle.

This paper is organized as follows. In Section 2, model description and some important preliminaries are given. In Section 3, the main result is presented.

\section{Model and Preliminaries}

Consider a neural network with time-varying delay, which can be described by the following equation:

$$
\begin{aligned}
\dot{x}_{i}(t)= & -C x_{i}(t)+A f\left(x_{i}(t)\right)+B f\left(x_{i}(t-\tau(t))\right) \\
& +c_{0} \sum_{j=1}^{N} d_{i j} \Gamma_{1} x_{j}(t) \quad(i=1,2, \cdots, N)
\end{aligned}
$$

$x_{i}(t)=\left(x_{i 1}(t), x_{i 2}(t), \cdots, x_{i n}(t)\right)^{\mathrm{T}} \in R^{n}$ is the state vector of the $i$-th node at time $t ; f: R^{n} \rightarrow R^{n}$ is a smooth vector function; $\tau(t)$ is the time varying delay; $C=\operatorname{diag}\left(c_{1}, c_{2}, \cdots, c_{n}\right) \in R^{n \times n}$ with $c_{i}>0(i=1,2, \cdots, n)$ denotes the state feedback coefficient matrix; $A=\left(a_{i j}\right) \in R^{n \times n}$ and $B=\left(b_{i j}\right) \in R^{n \times n}$ are respectively the weight and delayed weight matrices; $c_{0} \in R^{+}$is the network coupling 
strength; external coupling configuration matrix $D=\left(d_{i j}\right) \in R^{N \times N}$ represents the network topology and the coupling strength between nodes, the following conditions are met: if there is a connection between node $i$ and node $j$, then $d_{i j}>0$, if not $d_{i j}=0(i \neq j)$, and the diagonal element $d_{i i}=-\sum_{j=1, j \neq i}^{N} d_{i j}$, $i=1,2, \cdots, N$; matrix $\Gamma_{1} \in R^{n \times n}$ is a known constant positive definite diagonal matrix, which represents the internal coupling of the network.

We regard network (1) as a drive network, and the following equation is the corresponding response network:

$$
\begin{aligned}
\dot{y}_{i}(t)= & -C y_{i}(t)+A g\left(y_{i}(t)\right)+B g\left(y_{i}(t-\tau(t))\right) \\
& +c_{0} \sum_{j=1}^{N} g_{i j} \Gamma_{2} y_{j}(t)+u_{i} \quad(i=1,2, \cdots, N)
\end{aligned}
$$

$y_{i}(t)=\left(y_{i 1}(t), y_{i 2}(t), \cdots, y_{i n}(t)\right)^{\mathrm{T}} \in R^{n}$ is the state vector of the $i$-th node at time $t, g: R^{n} \rightarrow R^{n}$ is a smooth vector function; $G=\left(g_{i j}\right) \in R^{N \times N}, \Gamma_{2} \in R^{n \times n}$ are defined as $D=\left(d_{i j}\right) \in R^{N \times N}, \Gamma_{1} \in R^{n \times n}$ in network (1) respectively; $u_{i}$ is the synchronous controller to be designed.

We can see from the model of drive-response neural networks that the topology and node dynamics of the two networks can be different.

Definition 1 [25] The node error of generalized synchronization between systems (1) and (2) is defined as

$$
e_{i}(t)=y_{i}(t)-\varphi_{i}\left(x_{i}(t)\right), \quad i=1,2, \cdots, N
$$

where $\varphi_{i}: R^{n} \rightarrow R^{n}(i=1,2, \cdots, N)$ is a vector mapping.

If there is a controller $u_{i}$, such that $\lim _{t \rightarrow \infty} e_{i}(t)=0(i=1,2, \cdots, N)$, then the networks (1) and (2) are said to be generalized synchronized.

To get the generalized synchronization of networks (1) and (2), the controller $u_{i}$ is designed as follows:

$$
\begin{gathered}
u_{i}=J_{\varphi_{i}} \dot{x}_{i}(t)+C \varphi_{i}\left(x_{i}(t)\right)-A g\left(\varphi_{i}\left(x_{i}(t)\right)\right)-B g\left(\varphi_{i}\left(x_{i}(t-\tau(t))\right)\right) \\
-c_{0} \sum_{j=1}^{N} g_{i j} \Gamma_{2}\left(\varphi_{j}\left(x_{j}(t)\right)\right)-k_{i} e_{i}(t) \\
\dot{k}_{i}=r_{i}\left\|e_{i}(t)\right\|^{2}
\end{gathered}
$$

where $r_{i}$ is an arbitrary positive constant; $J_{\varphi_{i}}$ is the Jacobian matrix of the mapping $\varphi_{i}, \quad J_{\varphi_{i}}=\left|\begin{array}{cccc}\frac{\partial \varphi_{i 1}}{\partial x_{i 1}} & \frac{\partial \varphi_{i 1}}{\partial x_{i 2}} & \cdots & \frac{\partial \varphi_{i 1}}{\partial x_{i n}} \\ \frac{\partial \varphi_{i 2}}{\partial x_{i 1}} & \frac{\partial \varphi_{i 2}}{\partial x_{i 2}} & \cdots & \frac{\partial \varphi_{i 2}}{\partial x_{i n}} \\ \vdots & \vdots & \ddots & \vdots \\ \frac{\partial \varphi_{i n}}{\partial x_{i 1}} & \frac{\partial \varphi_{i n}}{\partial x_{i 2}} & \cdots & \frac{\partial \varphi_{i n}}{\partial x_{i n}}\end{array}\right|, i=1,2, \cdots, N$.

Under the action of the controller, the error dynamic equation of the system is described as 


$$
\begin{aligned}
\dot{e}_{i}(t)= & \dot{y}_{i}(t)-J_{\varphi_{i}} \dot{x}_{i}(t) \\
= & -C\left[y_{i}(t)-\varphi_{i}\left(x_{i}(t)\right)\right]+A\left[g\left(y_{i}(t)\right)-g\left(\varphi_{i}\left(x_{i}(t)\right)\right)\right] \\
& +B\left[g\left(y_{i}(t-\tau(t))\right)-g\left(\varphi_{i}\left(x_{i}(t-\tau(t))\right)\right)\right] \\
& +c_{0} \sum_{j=1}^{N} g_{i j} \Gamma_{2}\left[y_{j}(t)-\varphi_{j}\left(x_{j}(t)\right)\right]-k_{i} e_{i}(t)
\end{aligned}
$$

In order to study the problem, the following assumptions and lemma are needed.

Assumption 1 For function $g(x)$, there exists a positive that $L$ such that

$$
\|g(y)-g(x)\| \leq \sqrt{L}\|y-x\|,
$$

for any two vectors $x, y \in R^{n}$.

Assumption 2 The time varying delay $\tau(t)$ satisfies: $0 \leq \dot{\tau}(t) \leq \varepsilon<1$, where $\varepsilon$ is a known constant.

These two assumptions are very common in the synchronization of complex networks.

Lemma 1 [28] For any two vectors $x, y \in R^{n}$, the following formula holds $x^{\mathrm{T}} x+y^{\mathrm{T}} y \geq 2 x^{\mathrm{T}} y$.

\section{Main Result}

In this section, we investigate the generalized synchronization problem of networks (1) and (2). The main result is given by the following theorem.

Theorem 1 If the topological structures of the drive-response neural networks (1) and (2) are known, then they can achieve generalized synchronization under the adaptive controller (4) and updated rule (5).

Proof: Construct the Lyapunov function as follows:

$$
V(t)=V_{1}(t)+V_{2}(t)
$$

where

$$
\begin{aligned}
& V_{1}(t)=\sum_{i=1}^{N} e_{i}^{\mathrm{T}}(t) e_{i}(t)+\sum_{i=1}^{N} \frac{1}{r_{i}}\left(k_{i}-\bar{k}\right)^{2} \\
& V_{2}(t)=\sum_{i=1}^{N} \frac{\alpha}{1-\varepsilon} \int_{t-\tau(t)}^{t} e_{i}^{\mathrm{T}}(\theta) e_{i}(\theta) \mathrm{d} \theta
\end{aligned}
$$

$\bar{k}$ and $\alpha$ are positive constants to be determined.

Calculating the time derivative of $V(t)$ along the error system (6),

$$
\dot{V}(t)=\dot{V}_{1}(t)+\dot{V}_{2}(t)
$$

According to (5) and (6), we can get

$$
\begin{aligned}
\dot{V}_{1}(t)= & 2 \sum_{i=1}^{N} e_{i}^{\mathrm{T}}(t) \dot{e}_{i}(t)+2 \sum_{i=1}^{N} \frac{1}{r_{i}}\left(k_{i}-\bar{k}\right) \dot{k}_{i} \\
= & -2 \sum_{i=1}^{N} e_{i}^{\mathrm{T}}(t) C\left[y_{i}(t)-\varphi_{i}\left(x_{i}(t)\right)\right]-2 \sum_{i=1}^{N} \bar{k}\left\|e_{i}(t)\right\|^{2} \\
& +2 \sum_{i=1}^{N} e_{i}^{\mathrm{T}}(t) A\left[g\left(y_{i}(t)\right)-g\left(\varphi_{i}\left(x_{i}(t)\right)\right)\right]
\end{aligned}
$$




$$
\begin{aligned}
& +2 \sum_{i=1}^{N} e_{i}^{\mathrm{T}}(t) B\left[g\left(y_{i}(t-\tau(t))\right)-g\left(\varphi_{i}\left(x_{i}(t-\tau(t))\right)\right)\right] \\
& +2 c_{0} \sum_{i=1}^{N} \sum_{j=1}^{N} e_{i}^{\mathrm{T}}(t) g_{i j} \Gamma_{2}\left[y_{j}(t)-\varphi_{j}\left(x_{j}(t)\right)\right] \\
& =W_{1}+W_{2}+W_{3}+W_{4}
\end{aligned}
$$

In the above equation,

$$
\begin{aligned}
& W_{1}=-2 \sum_{i=1}^{N} e_{i}^{\mathrm{T}}(t) C\left[y_{i}(t)-\varphi_{i}\left(x_{i}(t)\right)\right]-2 \sum_{i=1}^{N} \bar{k}\left\|e_{i}(t)\right\|^{2}, \\
& W_{2}=2 \sum_{i=1}^{N} e_{i}^{\mathrm{T}}(t) A\left[g\left(y_{i}(t)\right)-g\left(\varphi_{i}\left(x_{i}(t)\right)\right)\right], \\
& W_{3}=2 \sum_{i=1}^{N} e_{i}^{\mathrm{T}}(t) B\left[g\left(y_{i}(t-\tau(t))\right)-g\left(\varphi_{i}\left(x_{i}(t-\tau(t))\right)\right)\right], \\
& W_{4}=2 c_{0} \sum_{i=1}^{N} \sum_{j=1}^{N} e_{i}^{\mathrm{T}}(t) g_{i j} \Gamma_{2}\left[y_{j}(t)-\varphi_{j}\left(x_{j}(t)\right)\right] .
\end{aligned}
$$

Let $\lambda_{0}=\min \left(c_{1}, c_{2}, \cdots, c_{n}\right), e(t)=\left[e_{1}^{\mathrm{T}}(t), e_{2}^{\mathrm{T}}(t), \cdots, e_{N}^{\mathrm{T}}(t)\right]^{\mathrm{T}}$, then

$$
\begin{aligned}
W_{1} & =-2 \sum_{i=1}^{N} e_{i}^{\mathrm{T}}(t) C\left[y_{i}(t)-\varphi_{i}\left(x_{i}(t)\right)\right]-2 \sum_{i=1}^{N} \bar{k}\left\|e_{i}(t)\right\|^{2} \\
& =-2 \sum_{i=1}^{N} e_{i}^{\mathrm{T}}(t) C e_{i}(t)-2 \bar{k} \sum_{i=1}^{N} e_{i}^{\mathrm{T}}(t) e_{i}(t) \\
& \leq-2 \lambda_{0} \sum_{i=1}^{N} e_{i}^{\mathrm{T}}(t) e_{i}(t)-2 \bar{k} \sum_{i=1}^{N} e_{i}^{\mathrm{T}}(t) e_{i}(t) \\
& =-2\left(\lambda_{0}+\bar{k}\right) e^{\mathrm{T}}(t) e(t)
\end{aligned}
$$

By using Lemma 1 and Assumption 1, it's easy to get

$$
\begin{aligned}
W_{2} & =2 \sum_{i=1}^{N} e_{i}^{\mathrm{T}}(t) A\left[g\left(y_{i}(t)\right)-g\left(\varphi_{i}\left(x_{i}(t)\right)\right)\right] \\
\leq & \sum_{i=1}^{N} e_{i}^{\mathrm{T}}(t) A A^{\mathrm{T}} e_{i}(t) \\
& +\sum_{i=1}^{N}\left[g\left(y_{i}(t)\right)-g\left(\varphi_{i}\left(x_{i}(t)\right)\right)\right]^{\mathrm{T}}\left[g\left(y_{i}(t)\right)-g\left(\varphi_{i}\left(x_{i}(t)\right)\right)\right] \\
\leq & \lambda_{\max }\left(A A^{\mathrm{T}}\right) \sum_{i=1}^{N} e_{i}^{\mathrm{T}}(t) e_{i}(t)+L \sum_{i=1}^{N} e_{i}^{\mathrm{T}}(t) e_{i}(t) \\
& =\left(\lambda_{1}+L\right) e^{\mathrm{T}}(t) e(t)
\end{aligned}
$$

where $\lambda_{1}=\lambda_{\max }\left(A A^{\mathrm{T}}\right)$ is the maximum eigenvalue of matrix $A A^{\mathrm{T}}$.

Same as $W_{2}$, we have

$$
\begin{aligned}
W_{3} & =2 \sum_{i=1}^{N} e_{i}^{\mathrm{T}}(t) B\left[g\left(y_{i}(t-\tau(t))\right)-g\left(\varphi_{i}\left(x_{i}(t-\tau(t))\right)\right)\right] \\
\leq & \sum_{i=1}^{N} e_{i}^{\mathrm{T}}(t) B B^{\mathrm{T}} e_{i}(t)+\sum_{i=1}^{N}\left[g\left(y_{i}(t-\tau(t))\right)-g\left(\varphi_{i}\left(x_{i}(t-\tau(t))\right)\right)\right]^{\mathrm{T}} \\
& \times\left[g\left(y_{i}(t-\tau(t))\right)-g\left(\varphi_{i}\left(x_{i}(t-\tau(t))\right)\right)\right] \\
\leq & \lambda_{\max }\left(B B^{\mathrm{T}}\right) \sum_{i=1}^{N} e_{i}^{\mathrm{T}}(t) e_{i}(t)+L \sum_{i=1}^{N} e_{i}^{\mathrm{T}}(t-\tau(t)) e_{i}(t-\tau(t)) \\
& =\lambda_{2} e^{\mathrm{T}}(t) e(t)+L e^{\mathrm{T}}(t-\tau(t)) e(t-\tau(t))
\end{aligned}
$$


where $\lambda_{2}=\lambda_{\max }\left(B B^{\mathrm{T}}\right)$.

For convenience of presentation, let $M=G \otimes \Gamma_{2}, \quad \lambda_{3}=\lambda_{\max }\left(M M^{\mathrm{T}}\right)$, we obtain

$$
\begin{aligned}
W_{4}= & 2 c_{0} \sum_{i=1}^{N} \sum_{j=1}^{N} e_{i}^{\mathrm{T}}(t) g_{i j} \Gamma_{2}\left[y_{j}(t)-\varphi_{j}\left(x_{j}(t)\right)\right] \\
= & 2 c_{0} \sum_{i=1}^{N} \sum_{j=1}^{N} e_{i}^{\mathrm{T}}(t) g_{i j} \Gamma_{2} e_{j}(t) \\
= & 2 c_{0}\left[e_{1}^{\mathrm{T}}(t), e_{2}^{\mathrm{T}}(t), \cdots, e_{N}^{\mathrm{T}}(t)\right]\left|\begin{array}{cccc}
g_{11} \Gamma_{2} & g_{12} \Gamma_{2} & \cdots & g_{1 N} \Gamma_{2} \\
g_{21} \Gamma_{2} & g_{22} \Gamma_{2} & \cdots & g_{2 N} \Gamma_{2} \\
\vdots & \vdots & \ddots & \vdots \\
g_{N 1} \Gamma_{2} & g_{N 2} \Gamma_{2} & \cdots & g_{N N} \Gamma_{2}
\end{array}\right| \\
& \times\left[e_{1}(t), e_{2}(t), \cdots, e_{N}(t)\right]^{\mathrm{T}} \\
= & 2 c_{0} e^{\mathrm{T}}(t)\left(G \otimes \Gamma_{2}\right) e(t) \\
= & 2 c_{0} e^{\mathrm{T}}(t) M e(t) \\
\leq & c_{0}\left[e^{\mathrm{T}}(t) M M^{\mathrm{T}} e(t)+e^{\mathrm{T}}(t) e(t)\right] \\
\leq & c_{0}\left[\lambda_{\max }\left(M M^{\mathrm{T}}\right) e^{\mathrm{T}}(t) e(t)+e^{\mathrm{T}}(t) e(t)\right] \\
= & c_{0}\left(\lambda_{3}+1\right) e^{\mathrm{T}}(t) e(t)
\end{aligned}
$$

The time derivative of $V_{2}(t)$ can be expressed as

$$
\dot{V}_{2}(t)=\frac{\alpha}{1-\varepsilon} e^{\mathrm{T}}(t) e(t)-\frac{\alpha(1-\dot{\tau}(t))}{1-\varepsilon} e^{\mathrm{T}}(t-\tau(t)) e(t-\tau(t))
$$

Combining (7) - (15) yields

$$
\begin{aligned}
\dot{V}(t) \leq & -2 \lambda_{0} e^{\mathrm{T}}(t) e(t)+\left(L-\frac{\alpha(1-\dot{\tau}(t))}{1-\varepsilon}\right) e^{\mathrm{T}}(t-\tau(t)) e(t-\tau(t)) \\
& +\left(\lambda_{1}+L+\lambda_{2}+c_{0} \lambda_{3}+c_{0}-2 \bar{k}+\frac{\alpha}{1-\varepsilon}\right) e^{\mathrm{T}}(t) e(t)
\end{aligned}
$$

Take $\alpha=L, \bar{k}=\frac{1}{2}\left(\lambda_{1}+L+\lambda_{2}+c_{0} \lambda_{3}+c_{0}+\frac{L}{1-\varepsilon}+1\right)$, then under the premise of Assumption 2 that (16) can be estimated as

$$
\dot{V}(t) \leq-e^{\mathrm{T}}(t) e(t)
$$

It can be found from (17) that $0 \leq V(t) \leq V(0)$, this together with (7) and (8) signifies $V(t)$ is bounded. We can also obtain $\lim _{t \rightarrow \infty} \int_{t-\tau(t)}^{t} e^{\mathrm{T}}(\theta) e(\theta) \mathrm{d} \theta \leq V(0)-\lim _{t \rightarrow \infty} V(t)$. Based on Lyapunov stability theory and LaSalle's invariance principle [29], we have $\lim _{t \rightarrow \infty} e_{i}(t)=0(i=1,2, \cdots, N)$. Then it follows from Definition 1 that the drive-response networks (1) and (2) achieve generalized synchronization. The proof is completed.

\section{Conclusion}

In this paper, the problem of generalized synchronization for drive-response neural networks with time-varying delay and different node dynamics is concerned. 
We design an appropriate nonlinear adaptive controller and construct a suitable Lyapunov function so that the desired synchronization is achieved. Because generalized synchronization is a function mapping relationship, the controller of generalized synchronization is complex. How to simplify the controller and make it have a simpler form will be a problem to be studied in the future.

\section{Conflicts of Interest}

The author declares no conflicts of interest regarding the publication of this paper.

\section{References}

[1] Barabási, A.-L. and Albert, R. (1999) Emergence of Scaling in Random Networks. Science, 286, 509-512. https://doi.org/10.1126/science.286.5439.509

[2] Strogatz, S.H. (2001) Exploring Complex Networks. Nature, 410, 268-276. https://doi.org/10.1038/35065725

[3] Watts, D. and Strogatz, S. (1998) Collective Dynamics of "Small-World" Networks. Nature, 393, 440-442. https://doi.org/10.1038/30918

[4] Ott, E. (2002) Chaos in Dynamical Systems. Cambridge Univ. Press, Cambridge. https://doi.org/10.1017/CBO9780511803260

[5] Watts, M. (1999) Renormalization Group Analysis of the Small-World Network Model. Physics Letters A, 263, 341-346. https://doi.org/10.1016/S0375-9601(99)00757-4

[6] Newman, M. and Watts, D.J. (1999) Scaling and Percolation in the Small-World Network Model. Physical Review E, 60, 7332-7342. https://doi.org/10.1103/PhysRevE.60.7332

[7] Benson, A., Gleich, R.D.F. and Leskovec, J. (2016) Higher-Order Organization of Complex Networks. Science, 353, 163-166. https://doi.org/10.1126/science.aad9029

[8] Guo, W. (2011) Lag Synchronization of Complex Networks via Pinning Control. Nonlinear Analysis Real World Applications, 12, 2579-2585. https://doi.org/10.1016/j.nonrwa.2011.03.007

[9] Yu, C.B., Qin, J. and Gao, H. (2014) Cluster Synchronization in Directed Networks of Partial-State Coupled Linear Systems under Pinning Control. Automatica, 50, 2341-2349. https://doi.org/10.1016/j.automatica.2014.07.013

[10] Chung, S.J., Bandyopadhyay, S., Chang, I. and Hadaegh, F.Y. (2013) Phase Synchronization Control of Complex Networks of Lagrangian Systems on Adaptive Digraphs. Automatica, 49, 1148-1161. https://doi.org/10.1016/j.automatica.2013.01.048

[11] Wang, J.A. and Liu, H.P. (2010) Adaptive Synchronization between Two Different Complex Networks with Time-Varying Delay Coupling. Journal of University of Science and Technology Beijing, 32, 61-64.

[12] Arenas, A., Díaz-Guilera, A., Kurths, J., Moreno, Y. and Zhou, C. (2008) Synchronization in Complex Networks. Physics Reports, 18, Article ID: 037111.

[13] Radenković, M.S. and Krstić, M. (2018) Distributed Adaptive Consensus and Synchronization in Complex Networks of Dynamical Systems. Automatica, 91, 233 243. https://doi.org/10.1016/j.automatica.2018.01.039

[14] Yang, L.X. and Jiang, J. (2014) Adaptive Synchronization of Drive-Response Fractional-Order Complex Dynamical Networks with Uncertain Parameters. Communications in Nonlinear Science \& Numerical Simulation, 19, 1496-1506. https://doi.org/10.1016/j.cnsns.2013.09.021 
[15] Chen, X. and Lu, J. (2007) Adaptive Synchronization of Different Chaotic Systems with Fully Unknown Parameters. Physics Letters A, 364, 123-128. https://doi.org/10.1016/j.physleta.2006.11.092

[16] Yang, X., Cao, J. and Lu, J. (2012) Stochastic Synchronization of Complex Networks with Nonidentical Nodes via Hybrid Adaptive and Impulsive Control. IEEE Transactions on Circuits and Systems I Regular Papers, 59, 371-384. https://doi.org/10.1109/TCSI.2011.2163969

[17] Han, X., Lu, J.A. and Wu, X. (2008) Synchronization of Impulsively Coupled Systems. International Journal of Bifurcation and Chaos, 18, 1539-1549. https://doi.org/10.1142/S0218127408021154

[18] Qiang, S. and Cao, J. (2010) On Pinning Synchronization of Directed and Undirected Complex Dynamical Networks. Circuits and Systems I: Regular Papers, IEEE Transactions on Circuits and Systems I Regular Papers, 57, 672-680. https://doi.org/10.1109/TCSI.2009.2024971

[19] Deng, L., Wu, Z. and Wu, Q. (2013) Pinning Synchronization of Complex Network with Non-Derivative and Derivative Coupling. Nonlinear Dynamics, 73, 775-782. https://doi.org/10.1007/s11071-013-0830-y

[20] Razminia, A. and Baleanu, D. (2013) Complete Synchronization of Commensurate Fractional Order Chaotic Systems Using Sliding Mode Control. Mechatronics, 23, 873-879. https://doi.org/10.1016/j.mechatronics.2013.02.004

[21] Hu, M., Yang, Y., Xu, Z., Rong, Z. and Guo, L. (2007) Projective Synchronization in Drive-Response Dynamical Networks. Physica A: Statistical Mechanics and Its Applications, 381, 457-466. https://doi.org/10.1016/j.physa.2007.03.023

[22] Mei, S., Zeng, C.Y. and Tian, L.X. (2008) Projective Synchronization in Drive-Response Dynamical Networks of Partially Linear Systems with Time-Varying Coupling Delay. Physics Letters A, 372, 6904-6908. https://doi.org/10.1016/j.physleta.2008.10.019

[23] Shang, Y., Chen, M. and Kurths, J. (2009) Generalized Synchronization of Complex Networks. Physical Review E Statistical Nonlinear and Soft Matter Physics, 80, Article ID: 027201. https://doi.org/10.1103/PhysRevE.80.027201

[24] Wu, X., Wei, X.Z. and Jin, Z. (2009) Generalized Outer Synchronization between Complex Dynamical Networks. Chaos, 19, Article ID: 013109.

https://doi.org/10.1063/1.3072787

[25] Wang, J.A. (2012) Adaptive Generalized Synchronization between Two Different Complex Networks with Time-Varying Delay Coupling. Acta Physica Sinica, 61, Article ID: 020509. https://doi.org/10.7498/aps.61.020509

[26] Cao, J.D., Li, P. and Wang, W.W. (2006) Global Synchronization in Arrays of Delayed Neural Networks with Constant and Delayed Coupling. Physics Letters A, 353, 318-325. https://doi.org/10.1016/j.physleta.2005.12.092

[27] Langville, A.N. and Stewart, W.J. (2004) The Kronecker Product and Stochastic Automata Networks. Journal of Computational and Applied Mathematics, 167, 429447. https://doi.org/10.1016/j.cam.2003.10.010

[28] Liu, H., Lu, J.A. and Lu, J.H. (2009) Structure Identification of Uncertain General Complex Dynamical Networks with Time Delay. Automatica, 45, 1799-1807. https://doi.org/10.1016/j.automatica.2009.03.022

[29] Aeyels, D. (1995) Asymptotic Stability of Nonautonomous Systems by Lyapunov's Direct Method. Systems and Control Letters, 25, 273-280.

https://doi.org/10.1016/0167-6911(94)00088-D 